\title{
Navigation Performance of the Libration Point Satellite Navigation System in Cislunar Space
}

\author{
Lei Zhang and $\mathrm{Bo} \mathrm{Xu}$ \\ (School of Astronomy and Space Science, Nanjing University, Nanjing 210093, China) \\ (E-mail: xubo@nju.edu.cn)
}

\begin{abstract}
Based on the candidate architectures of the libration point satellite navigation system proposed in our previous work, a navigation performance study is conducted in this paper to verify the cislunar navigation ability of the proposed system. Using scalar satellite-to-satellite range measurement between the user and libration point navigation satellites, a virtual lunar exploration mission scenario is developed to verify the navigation performance of the candidate Earth-Moon $L_{1,2,4,5}$ four-satellite constellations. The simulation results indicate that the libration point satellite navigation system is available for cislunar navigation and the navigation accuracy of a few tens of metres can be achieved for both the trans-lunar cruise and lunar orbit phase. Besides that, it is also found that the navigation accuracy of the libration point satellite navigation system is sensitive to the orbit of the $L_{1}$ satellite. Once the $L_{1}$ navigation satellite is located in the Halo orbit or vertical Lyapunov orbit, the proposed system can present a better navigation performance in cislunar space.
\end{abstract}

\section{KEYWORDS}

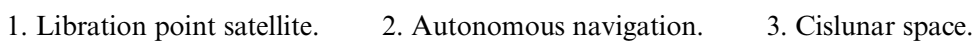

4. Numerical simulation.

Submitted: 12 April 2014. Accepted: 17 August 2014. First published online: 18 September 2014.

1. INTRODUCTION. In the past decade, more than ten lunar exploration missions have been launched to study the natural satellite of Earth, such as the European Space Agency's (ESA's) SMART-1 mission, the National Aeronautics and Space Administration's (NASA's) GRAIL mission, and China's Chang'e missions. The renewed interest in lunar exploration gives rise to an increasing demand for a high-precision navigation system in cislunar space. As the traditional approach that is based on extensive ground tracking is onerous and inadequate in some particular circumstances, an autonomous navigation scheme for lunar explorations is preferable.

The desire for autonomous cislunar navigation is a long-standing issue that has led to numerous studies since the 1960s. Keenan and Regenhardt (1962) proposed using star occultation measurements as an aid for navigation in cislunar space. Bowers (1966), followed by Tuckness and Young (1995) demonstrated the method that adopts angular measurements between known stars and known landmarks on the Earth or 
Moon for trans-lunar cruise navigation. Main advantages of these solutions lie in their autonomy and robustness, however, the navigation accuracy may not satisfy the requirement of actual missions (Christian and Lightsey, 2009). Considering the specific dynamical properties of the Earth-Moon system, Farquhar (1967) first proposed the concept of using Earth-Moon libration point satellites for lunar navigation. Due to strong asymmetry of the three-body force field, a spacecraft located in the Libration Point Orbit (LPO) can track other spacecraft using crosslink range measurements and determine the absolute positions of both spacecraft simultaneously without any mathematical constraints. This linked, autonomous orbit determination method is known as Liaison navigation which was first proposed by Hill et al. (2005) and then followed by many other researchers. Parker et al. (2013) and Leonard et al. (2013) proved that by adding a single navigation satellite located around the Earth-Moon $L_{1}$ or $L_{2}$ libration point, the number of active ground tracking stations can be greatly reduced for a lunar exploration mission and the navigation accuracy can also be improved. Hill himself has also completed a systematic study on the autonomous orbit determination for LPOs in his PhD dissertation (Hill, 2007).

Zhang and $\mathrm{Xu}$ (2014) proposed a concept of using the different libration point orbits to construct the Universe Lighthouse for cislunar navigation, where some candidate libration point satellite navigation architectures are obtained based on comprehensive consideration of constellation coverage ability and autonomous orbit determination performance. As a subsequent work of the libration point satellite navigation system design, this paper is focused on navigation performance analysis in cislunar space, specifically the trans-lunar cruise and lunar orbit phase. With the aid of the extended Kalman filter, the satellite-to-satellite range tracking data between the user spacecraft and libration point navigation satellites is used to autonomously determine the orbit of the user spacecraft, and no ground-based measurements are involved in the navigation process. The navigation performance of different EarthMoon $L_{1,2,4,5}$ four-satellite constellations are verified by numerical simulations.

The remainder of this paper is organised as follows. In Section 2, a brief review of the candidate architectures of the libration point satellite navigation system is given. After describing the mission scenario considered in this paper, the spacecraft dynamic model and observation measurements are explained in Section 3 and Section 4 gives a detailed description of the algorithm and state noise compensation method adopted in the filter process. After that, cislunar navigation simulations are performed for both the trans-lunar cruise phase and lunar orbit phase in Section 5. Finally, some brief conclusions are drawn in Section 6.

\section{CANDidATE NAVIGATION ARCHITECTURES. Libration} point orbits around the Earth-Moon equilibrium points are promising candidates to build navigation architectures in cislunar space, which has been proved by many researchers. With the aid of the Liaison technique, the autonomy of the libration point satellite can be guaranteed and an autonomous satellite navigation system can thus be founded. An architecture analysis study was completed in our previous work and some feasible three-satellite and four-satellite navigation constellations were obtained to achieve continuous global coverage for lunar orbits, with the latter having a better coverage performance. Therefore, in the following discussions, the Earth-Moon $L_{1,2,4,5}$ four-satellite constellations are selected as the focus of the cislunar navigation 
Table 1. Candidate navigation architectures of the Earth-Moon $L_{1,2,4,5}$ four-satellite constellation.

\begin{tabular}{llllll}
\hline ID & Constellation Type & LPO at L1 & LPO at L2 & LPO at L4 & LPO at L5 \\
\hline 1 & Halo-Halo-VP-VP & $A_{H} \leqslant 11603 \mathrm{~km}$ & $A_{H} \leqslant 18 \mathrm{~km}$ & $A_{V P} \leqslant 106 \mathrm{~km}$ & $A_{V P} \leqslant 106 \mathrm{~km}$ \\
2 & PL-Halo-VP-VP & $A_{P L} \leqslant 5801 \mathrm{~km}$ & $A_{H} \leqslant 18 \mathrm{~km}$ & $A_{V P} \leqslant 106 \mathrm{~km}$ & $A_{V P} \leqslant 106 \mathrm{~km}$ \\
3 & VL-Halo-VP-VP & $A_{V L} \leqslant 8702 \mathrm{~km}$ & $A_{H} \leqslant 18 \mathrm{~km}$ & $A_{V P} \leqslant 106 \mathrm{~km}$ & $A_{V P} \leqslant 106 \mathrm{~km}$ \\
4 & Halo-PL-VP-VP & $A_{H} \leqslant 11603 \mathrm{~km}$ & $A_{P L} \leqslant 11225 \mathrm{~km}$ & $A_{V P} \leqslant 148 \mathrm{~km}$ & $A_{V P} \leqslant 148 \mathrm{~km}$ \\
5 & PL-PL-VP-VP & $A_{P L} \leqslant 5801 \mathrm{~km}$ & $A_{P L} \leqslant 11225 \mathrm{~km}$ & $A_{V P} \leqslant 148 \mathrm{~km}$ & $A_{V P} \leqslant 148 \mathrm{~km}$ \\
6 & VL-PL-VP-VP & $A_{V L} \leqslant 8702 \mathrm{~km}$ & $A_{P L} \leqslant 11225 \mathrm{~km}$ & $A_{V P} \leqslant 148 \mathrm{~km}$ & $A_{V P} \leqslant 148 \mathrm{~km}$ \\
7 & Halo-VL-VP-VP & $A_{H} \leqslant 11603 \mathrm{~km}$ & $A_{V L} \leqslant 26 \mathrm{~km}$ & $A_{V P} \leqslant 135 \mathrm{~km}$ & $A_{V P} \leqslant 135 \mathrm{~km}$ \\
8 & PL-VL-VP-VP & $A_{P L} \leqslant 5801 \mathrm{~km}$ & $A_{V L} \leqslant 26 \mathrm{~km}$ & $A_{V P} \leqslant 135 \mathrm{~km}$ & $A_{V P} \leqslant 135 \mathrm{~km}$ \\
9 & VL-VL-VP-VP & $A_{V L} \leqslant 8702 \mathrm{~km}$ & $A_{V L} \leqslant 26 \mathrm{~km}$ & $A_{V P} \leqslant 135 \mathrm{~km}$ & $A_{V P} \leqslant 135 \mathrm{~km}$ \\
\hline
\end{tabular}

performance study and the candidate four-satellite navigation architectures are listed in Table 1 (Zhang and $\mathrm{Xu}, 2014$ ).

Candidate orbits for these navigation architectures are: Halo orbits (Halo), Planar Lyapunov orbits (PL) and Vertical Lyapunov orbits (VL) around the collinear libration points and Vertical Periodic orbits (VP) around the triangular libration points. The variables $A_{H}, A_{P L}, A_{V L}$ and $A_{V P}$ in the table are amplitude parameters which will be used to compute the LPOs. The construction details of these orbits can be found in Lei et al. (2013a) and Lei and Xu (2013b).

Since the autonomous orbit determination performance of the Earth-Moon $L_{1,2,4,5}$ four-satellite constellation has been verified by the Liaison technique in our previous work and it was found that the maximum position determination error in a 180 days period can be controlled within a few metres, positions of the libration point navigation satellites will be considered as known with an implicit error and their broadcast ephemeris will be used as given parameters in the navigation simulation process.

3. MISSION SCENARIOS AND MODELS. Before we start the cislunar navigation simulation, a reference mission scenario must first be developed. This section will cover a detailed description of the lunar exploration mission, as well as the spacecraft dynamic model and observation measurement model.

3.1. Design reference mission. The reference mission considered in this work mainly consists of two operation phases: a trans-lunar cruise phase and lunar orbit phase. The trans-lunar cruise departs from a LEO parking orbit with the orbital parameters given in Table 2.

The initial epoch of the mission is defined as 1 January 2025 UTC. By performing an approximately $3.12 \mathrm{~km} / \mathrm{s}$ manoeuvre in the along-track direction, the spacecraft is inserted into the trans-lunar cruise trajectory at 1 Jan 2025 08:03:05 UTC. After approximately three days of cruise, the spacecraft reaches perilune at 4 January 2025 07:48:11 UTC with an altitude of $444 \mathrm{~km}$. Then a Lunar Orbit Insertion (LOI) manoeuvre is performed to insert the spacecraft into a circular, polar lunar orbit. The magnitude of the LOI is approximately $854 \mathrm{~m} / \mathrm{s}$ and the orbital parameters of the final lunar orbit are summarised in Table 3.

One or more trajectory correction manoeuvres may be needed to prune the trajectory and target the final nominal orbit in real missions; this is not considered here for simplicity, which will not have any significant effect on the following simulation results. 
Table 2. Orbital parameters of the initial LEO parking orbit.

\begin{tabular}{lccccc}
\hline Epoch & $a(\mathrm{~km})$ & $e$ & $i\left(^{\circ}\right)$ & $\Omega\left({ }^{\circ}\right)$ & $\omega+f\left({ }^{\circ}\right)$ \\
\hline 1 Jan 2025 04:50:21 UTC & 6675 & 0.00 & 28.09 & 3.05 & $116 \cdot 02$ \\
\hline
\end{tabular}

Table 3. Orbital parameters of the lunar orbit.

\begin{tabular}{lccccr}
\hline Epoch & $a(\mathrm{~km})$ & $e$ & $i\left(^{\circ}\right)$ & $\Omega\left({ }^{\circ}\right)$ & $\omega+f\left({ }^{\circ}\right)$ \\
\hline 4 Jan 2025 10:21:28 UTC & 2190 & 0.00 & 89.89 & $288 \cdot 89$ & $307 \cdot 85$ \\
\hline
\end{tabular}

3.2. Spacecraft dynamic model. The spacecraft dynamic models of the two operation phases above are different, but both can be formulated as a perturbed twobody problem. The perturbed equation of motion can be written in a general form as

$$
\ddot{\boldsymbol{r}}=-\frac{\mu}{r^{3}} \boldsymbol{r}+\boldsymbol{F}_{\varepsilon}
$$

where $\boldsymbol{r}$ is the position vector of the spacecraft, $\mu$ is the gravitational parameter of the central body and $\boldsymbol{F}_{\varepsilon}$ is the resultant vector of all the perturbing accelerations.

For the trans-lunar cruise phase, the motion of the spacecraft is studied in the International Celestial Reference Frame (ICRF). Perturbations considered include the non-spherical gravitation of the Earth, the third body perturbations of the Moon, the Sun and planets, and the non-gravitational perturbation due to solar radiation pressure. The non-spherical gravitational potential of the Earth can be commonly written as

$$
\Delta V=\frac{\mu}{R} \sum_{l \geqslant 2} \sum_{m=0}^{l}\left(\frac{a_{e}}{R}\right)^{l} \bar{P}_{l m}(\sin \varphi)\left[\bar{C}_{l m} \cos m \lambda_{G}+\bar{S}_{l m} \sin m \lambda_{G}\right]
$$

where $a_{e}$ is the equatorial radius of the Earth, $\bar{P}_{l m}$ is the normalized associated Legendre polynomial, and $\bar{C}_{l m}$ and $\bar{S}_{l m}$ are spherical harmonics related to the nonspherical gravitational potential. In this work, the WGS84 gravity field model is adopted for the non-spherical Earth (Defense Mapping Agency, 1984). As the gravitational potential is expressed in the central body fixed frame, a coordinate transformation is also needed to convert the acceleration from the body fixed frame to the inertial frame. After considering these, the non-spherical gravitational acceleration can then be obtained by

$$
\boldsymbol{F}_{N S P}=\left(\frac{\partial \Delta V}{\partial \boldsymbol{r}}\right)^{T}=\left(\frac{\partial \boldsymbol{R}}{\partial \boldsymbol{r}}\right)^{T}\left(\frac{\partial \Delta V}{\partial \boldsymbol{R}}\right)^{T}
$$

where $(\partial \boldsymbol{R} / \partial \boldsymbol{r})^{T}$ is the conversion from the International Terrestrial Reference Frame (ITRF) to ICRF, and the International Astronomical Union's Standards of Fundamental Astronomy (IAU SOFA) software collection can be conveniently adopted to finish this transformation (IAU SOFA Board, 2010).

The acceleration due to the third bodies can be written in a simple form as

$$
\boldsymbol{F}_{T B P}=-\mu^{\prime}\left(\frac{\Delta}{\Delta^{3}}+\frac{\boldsymbol{r}^{\prime}}{r^{\prime 3}}\right)
$$


where $\mu^{\prime}$ is the gravitational parameter of the third body, here for the trans-lunar cruise, third bodies considered include the Moon, the Sun and the eight planets except the Earth. $\Delta$ is the position vector from the third body to the spacecraft, and $\boldsymbol{r}^{\prime}$ is the position vector from the Earth to the third body. The positions of these celestial bodies can be obtained from the JPL DE405 ephemerides (Standish, 1998).

As the spacecraft is far away from the Earth, influence of solar radiation pressure should also be considered. Based on a cylindrical shadow model, the acceleration due to solar radiation pressure can be written as

$$
\boldsymbol{F}_{S R P}=v C_{R}\left(\frac{S}{m}\right) \rho_{\boldsymbol{e}}\left(\frac{1 A U}{r_{\boldsymbol{e}}}\right)^{2} \frac{\boldsymbol{r}_{\boldsymbol{e}}}{r_{\boldsymbol{e}}}
$$

where $\boldsymbol{r}_{\boldsymbol{e}}$ is the position vector from the Sun to the spacecraft, $\rho_{\boldsymbol{e}}=4.56 \times 10^{-6} \mathrm{~N} / \mathrm{m}^{2}$ is the solar radiation pressure constant in the vicinity of Earth, $(S / m)$ is the area-mass ratio of the spacecraft and $C_{R}$ is the reflectivity coefficient with a value of approximately one. The eclipse factor $v$ is determined by the following relations:

$$
\left\{\begin{array}{l}
\text { Earth shadow : } \cos \psi_{1}=\left(\frac{\boldsymbol{r}}{r}\right) \cdot\left(\frac{\boldsymbol{r}_{s}}{r_{s}}\right)<0 \& \sin \psi_{1}=\sqrt{1-\cos ^{2} \psi_{1}}<\frac{a_{e}}{r} \\
\text { Moon shadow : } \cos \psi_{2}=\left(\frac{\boldsymbol{r}-\boldsymbol{r}_{m}}{\left|\boldsymbol{r}-\boldsymbol{r}_{m}\right|}\right) \cdot\left(\frac{\boldsymbol{r}_{s}}{r_{s}}\right)<0 \& \sin \psi_{2}=\sqrt{1-\cos ^{2} \psi_{2}}<\frac{a_{m}}{\left|\boldsymbol{r}-\boldsymbol{r}_{m}\right|}
\end{array}\right.
$$

$\boldsymbol{r}_{s}$ and $\boldsymbol{r}_{m}$ in the above relations represent the geocentric position vector of the Sun and the Moon, while $a_{e}$ and $a_{m}$ are the equatorial radiuses of the Earth and the Moon, respectively. Once one of the above relations is satisfied, the eclipse factor $v=0$, which means the spacecraft is in the shadow of the Earth or the Moon, else $v=1$ meaning that the spacecraft is in sunlight.

After the spacecraft arrives at the Moon, the reference frame used to study the motion will be changed from the ICRF to the Moon-Centred Inertial (MCI) frame. The dynamical model of the spacecraft is also different from the previous phase, since the central body has been changed to the Moon. The perturbations considered are similar, but the gravitation of the Earth now becomes a third-body perturbation. Besides that, the solid tide perturbation should also be considered for the lunar orbit phase.

The lunar gravity field adopted in this work is the LP165 model (Konopliv et al., 2001). In order to obtain the conversion matrix $(\partial \boldsymbol{R} / \partial \boldsymbol{r})^{T}$ in Equation (3), a two-step transformation is considered. First is the transformation from the Moon-Centred Moon-Fixed (MCMF) frame to ICRF, which can be obtained from the lunar libration parameters as follows

$$
\boldsymbol{R}_{M C M F}=[M] \boldsymbol{r}_{I C R S},[M]=R_{Z}(\Lambda) R_{X}\left(i_{S}\right) R_{Z}\left(\Omega^{\prime}\right)
$$

$\Omega^{\prime}, i_{S}$ and $\Lambda$ are the Euler angles given by the JPL DE405 ephemeris. Then the conversion from the ICRF to the MCI frame can be performed by

$$
\boldsymbol{r}_{I C R S}=[N]^{T} \boldsymbol{r}_{M C I},[N]=R_{Z}\left(-\Omega_{m}\right) R_{X}\left(-I_{m}\right) R_{Z}\left(\Omega_{m}\right) R_{X}(\varepsilon)
$$

where $\Omega_{m}$ is the mean longitude of the ascending node of the Moon's orbit referred to the ecliptic, $I_{m}$ is the inclination of the mean lunar equator to the ecliptic and $\varepsilon$ is the mean obliquity of the ecliptic. Values of these angles can be obtained from the Astronomical Almanac (US Nautical Almanac Office, 2013). 
The tidal deformation of the Moon will cause temporal variations in the gravitational potential, which in turn will introduce perturbations in the motion of close satellites. Previous research found that the maximum term of solid tide perturbation may reach the same order of magnitude as the third-body perturbation of the Sun for a low lunar orbit (Liu and Wang, 2006). Thus it needs to be considered in the lunar orbit phase. The maximum variation of the lunar gravitational potential caused by tidal deformation can be written in the form

$$
\Delta V_{2,0}=\frac{\mu_{e}}{r_{e}^{3}} \frac{k_{2}}{r^{3}} P_{2}\left(\cos \varphi_{e}\right)
$$

where $\mu_{e}$ is the gravitational parameter of the Earth, $r_{e}$ is the distance between the Earth and the Moon, $k_{2}=0.029966$ is the second order Love number of the Moon and $P_{2}$ is the second order Legendre polynomial. The lunicentric angle $\varphi_{e}$ between the spacecraft and the Earth can be computed from the following relation,

$$
\cos \varphi_{e}=\left(\frac{\boldsymbol{r}}{r}\right) \cdot\left(\frac{\boldsymbol{r}_{e}}{r_{e}}\right)
$$

Then the solid tide perturbation can be obtained directly by the potential gradient

$$
\boldsymbol{F}_{S T P}=\left(\frac{\partial \Delta V_{2,0}}{\partial \boldsymbol{r}}\right)^{T}
$$

Other perturbations like the third body perturbation and the solar radiation pressure perturbation are similar to the trans-lunar cruise phase, except the origin has been changed to the Moon and position vectors are now expressed in a Moon-centred frame.

3.3. Observation measurement model. The observation model adopted in this work is an idealised range measurement between the spacecraft and libration point navigation satellites. Once a navigation satellite is visible to the user spacecraft, idealised satellite-to-satellite range data is obtained by the user, which can be defined in the following form

$$
\rho=\sqrt{\left(x-x_{L}\right)^{2}+\left(y-y_{L}\right)^{2}+\left(z-z_{L}\right)^{2}}+\sigma_{\text {bias }}+\sigma_{\text {noise }}
$$

where $\boldsymbol{r}=(x, y, z)$ is the position vector of the user and $\boldsymbol{r}_{L}=\left(x_{L}, y_{L}, z_{L}\right)$ is the position vector of the libration point satellite. As various errors exist in every observation measurement, two main components are considered in the above expression. One is the systematic bias $\sigma_{\text {bias }}$, which is related to the measurement model, and the other is a random white noise $\sigma_{\text {noise }}$ that differs in every measurement. Both of them are drawn from Gaussian distributions with zero mean and the given standard deviations.

Noting that the position of the libration point satellite is generally expressed in the Earth-Moon barycentric synodic frame, a coordinate transformation is needed to transform it to the same reference frame as the user spacecraft. The conversion from the Earth-Moon barycentric synodic frame to the ICRF can be finished by a simple translation and rotation, given by Equation (13)

$$
\boldsymbol{r}_{L}=[C]\left(\boldsymbol{r}_{L}^{\prime}+\left[\mathrm{d}_{E C}, 0,0\right]^{T}\right)
$$

where $\boldsymbol{r}_{L}^{\prime}$ is the position vector of the libration point satellite expressed in the synodic frame, $\mathrm{d}_{E C}$ is the distance between the Earth and the Earth-Moon barycentre, and $[C]$ is a rotation matrix which can be defined by the position and velocity vectors of the 
Moon as follows

$$
[C]=\left[\hat{\boldsymbol{e}}_{1}, \hat{\boldsymbol{e}}_{2}, \hat{\boldsymbol{e}}_{3}\right], \hat{\boldsymbol{e}}_{1}=\frac{\boldsymbol{r}_{m}}{\left|\boldsymbol{r}_{m}\right|}, \hat{\boldsymbol{e}}_{3}=\frac{\boldsymbol{r}_{m} \times \dot{\boldsymbol{r}}_{m}}{\left|\boldsymbol{r}_{m} \times \dot{\boldsymbol{r}}_{m}\right|}, \hat{\boldsymbol{e}}_{2}=\hat{\boldsymbol{e}}_{3} \times \hat{\boldsymbol{e}}_{1}
$$

While for the conversion from the Earth-Moon barycentric synodic frame to the MCI frame, it can be finished by a similar process as

$$
\boldsymbol{r}_{L}=[N][C]\left(\boldsymbol{r}_{L}^{\prime}-\left[\mathrm{d}_{M C}, 0,0\right]^{T}\right)
$$

where $\mathrm{d}_{M C}$ is the distance between the Moon and the Earth-Moon barycentre. The definitions of $[N]$ and $[C]$ are the same as in the previous case.

Using the nominal orbits of the user and libration point satellites, observation measurements are simulated according to the above description. Then an Extended Kalman Filter (EKF) algorithm is adopted to finish the navigation performance analysis.

4. NAVIGATION FILTER ALGORITHM. The EKF is one of the most common methods used in real-time navigation, and it is also adopted in this work. The state vector being estimated consists of the position and velocity of the spacecraft

$$
\boldsymbol{X}=[x, y, z, \dot{x}, \dot{y}, \dot{z}]^{T}
$$

which is governed by a system of nonlinear differential equations, denoted by

$$
\dot{\boldsymbol{X}}=F(\boldsymbol{X}, t)+\boldsymbol{G}(t)
$$

where $F(\boldsymbol{X}, t)$ is the known term that has been discussed in the previous section, $\boldsymbol{G}(t)$ is the model bias compensation and assumed to be a white noise process with

$$
E[\boldsymbol{G}(t)]=0 \text { and } E\left[\boldsymbol{G}(t) \boldsymbol{G}^{T}(\tau)\right]=Q(t) \delta(t-\tau)
$$

where $\delta(t-\tau)$ is the Dirac delta function, and $Q$ is the process noise covariance matrix which will be discussed later in this section.

Since the expected value of the process noise is zero, it does not affect the orbit on average. Therefore the state estimate can be propagated as usual without consideration of the noise term. But the time update equation for the state error covariance is changed due to the presence of process noise. The modified covariance propagation formula can be obtained with the state transition matrix $\Phi$ in the following way (Montenbruck and Gill, 2000)

$$
\bar{P}_{i}=\Phi\left(t_{i}, t_{i-1}\right) P_{i-1} \Phi^{T}\left(t_{i}, t_{i-1}\right)+\int_{t_{i-1}}^{t_{i}} \Phi\left(t_{i}, \tau\right) Q(\tau) \Phi^{T}\left(t_{i}, \tau\right) \mathrm{d} \tau
$$

the state transition matrix is obtained by integrating

$$
\dot{\Phi}\left(t, t_{i}\right)=A(t) \Phi\left(t, t_{i}\right)
$$

with the initial condition $\Phi\left(t_{i}, t_{i}\right)=I$, and

$$
A(t)=\frac{\partial F\left(\boldsymbol{X}^{*}, t\right)}{\partial \boldsymbol{X}(t)}
$$

where $*$ means the partial derivative is evaluated along the reference trajectory. 
Using the observation data at time $t_{i}$, a measurement update for the state and covariance matrix can then be computed by the following relations (Tapley et al., 2004)

$$
\begin{gathered}
K_{i}=\bar{P}_{i} \tilde{H}_{i}^{T}\left[\tilde{H}_{i} \bar{P}_{i} \tilde{H}_{i}^{T}+R_{i}\right]^{-1} \\
\hat{\boldsymbol{X}}_{i}=\boldsymbol{X}_{i}^{*}+K_{i} \boldsymbol{y}_{i} \\
P_{i}=\left[I-K_{i} \tilde{H}_{i}\right] \bar{P}_{i}
\end{gathered}
$$

where $K_{i}$ is called the Kalman gain, $R_{i}$ is the observation covariance matrix, $\mathbf{y}_{i}$ is the observation deviation, and $\tilde{H}_{i}$ is the observation-state mapping matrix. Here for the scalar satellite-to-satellite range data, $\tilde{H}_{i}$ is computed using the current state as

$$
\tilde{H}_{i}=\frac{\partial \rho\left(\boldsymbol{X}^{*}, t_{i}\right)}{\partial \boldsymbol{X}}=\left[\frac{x_{i}^{*}-x_{L}^{i}}{\rho_{i}}, \frac{y_{i}^{*}-y_{L}^{i}}{\rho_{i}}, \frac{z_{i}^{*}-z_{L}^{i}}{\rho_{i}}, 0,0,0\right]
$$

The newly updated state is then propagated forward to the time of next observation and the time and measurement updates are repeated. With regard to the above discussions, two additional remarks should be made. One is the computation of the Jacobian matrix $A(t)$, which can be simplified due to the characteristics of the filter, and the other is the method that is adopted to determine the process noise covariance matrix $Q(t)$.

The Jacobian matrix $A(t)$ can cause cumbersome analytic expressions when using a complex force model. One solution to avoid this problem is using a truncated force model to compute the Jacobian matrix, by noting the gradual convergence property of the filter process. For the trans-lunar cruise phase, only the Earth point-mass gravitation is considered in the Jacobian matrix computation, which results in

$$
A(t)=\left[\begin{array}{cccccc}
0 & 0 & 0 & 1 & 0 & 0 \\
0 & 0 & 0 & 0 & 1 & 0 \\
0 & 0 & 0 & 0 & 0 & 1 \\
-\frac{\mu_{e}}{r^{3}}+\frac{3 \mu_{e} x^{2}}{r^{5}} & \frac{3 \mu_{e} x y}{r^{5}} & \frac{3 \mu_{e} x z}{r^{5}} & 0 & 0 & 0 \\
\frac{3 \mu_{e} x y}{r^{5}} & -\frac{\mu_{e}}{r^{3}}+\frac{3 \mu_{e} y^{2}}{r^{5}} & \frac{3 \mu_{e} y z}{r^{5}} & 0 & 0 & 0 \\
\frac{3 \mu_{e} x z}{r^{5}} & \frac{3 \mu_{e} y z}{r^{5}} & -\frac{\mu_{e}}{r^{3}}+\frac{3 \mu_{e} z^{2}}{r^{5}} & 0 & 0 & 0
\end{array}\right]
$$

While for the Lunar orbit phase, the $J_{2}$ term of Lunar non-spherical gravitation is added and the Jacobian matrix $A(t)$ has the form

$$
A(t)=\left[\begin{array}{cccccc}
0 & 0 & 0 & 1 & 0 & 0 \\
0 & 0 & 0 & 0 & 1 & 0 \\
0 & 0 & 0 & 0 & 0 & 1 \\
c_{1}+c_{2} x^{2} & c_{2} x y & c_{2} x z & 0 & 0 & 0 \\
c_{2} x y & c_{1}+c_{2} y^{2} & c_{2} y z & 0 & 0 & 0 \\
\left(c_{2}+c_{3}\right) x z & \left(c_{2}+c_{3}\right) y z & c_{1}+\left(c_{2}+c_{3}\right) z^{2} & 0 & 0 & 0
\end{array}\right]
$$


With

$$
\begin{aligned}
& c_{1}=-\frac{\mu_{m}}{r^{3}}+\frac{3 J_{2}}{2}\left(\frac{5 z^{2}}{r^{7}}-\frac{1}{r^{5}}\right) \\
& c_{2}=\frac{3 \mu_{m}}{r^{5}}+\frac{3 J_{2}}{2}\left(-\frac{35 z^{2}}{r^{9}}+\frac{5}{r^{7}}\right) \\
& c_{3}=\frac{15 J_{2} z}{r^{7}}
\end{aligned}
$$

where $\mu_{m}$ is the gravitational parameter of the Moon and $J_{2}=-\sqrt{5} \bar{C}_{2,0}=2.03227 \times$ $10^{-4}$.

The process noise covariance matrix $Q(t)$ is used to compensate for the unmodelled accelerations and stop the filter from getting insensitive to further observations. The question of how to determine the process noise is complex. In this work, $Q(t)$ is chosen as a diagonal matrix such as

$$
Q(t)=\left[\begin{array}{cccccc}
\sigma_{x}^{2} & 0 & 0 & 0 & 0 & 0 \\
0 & \sigma_{y}^{2} & 0 & 0 & 0 & 0 \\
0 & 0 & \sigma_{z}^{2} & 0 & 0 & 0 \\
0 & 0 & 0 & \sigma_{\dot{x}}^{2} & 0 & 0 \\
0 & 0 & 0 & 0 & \sigma_{\dot{y}}^{2} & 0 \\
0 & 0 & 0 & 0 & 0 & \sigma_{\dot{z}}^{2}
\end{array}\right]
$$

and it is further assumed that the velocity of the spacecraft can be considered as constant between two contiguous observations, which leads the state transition matrix $\Phi\left(t_{i}, \tau\right)$ to be

$$
\Phi\left(t_{i}, \tau\right)=\left[\begin{array}{cc}
\boldsymbol{I} & \left(t_{i}-\tau\right) \boldsymbol{I} \\
0 & \boldsymbol{I}
\end{array}\right]
$$

Then the integral in Equation (19) can be computed analytically and yields

$$
\begin{aligned}
& \int_{t_{i-1}}^{t_{i}} \Phi\left(t_{i}, \tau\right) Q(\tau) \Phi^{T}\left(t_{i}, \tau\right) \mathrm{d} \tau \\
& {\left[\begin{array}{cccccc}
\sigma_{x}^{2} \Delta t+\sigma_{\dot{x}}^{2} \frac{\Delta t^{3}}{3} & 0 & 0 & \sigma_{\dot{x}}^{2} \frac{\Delta t^{2}}{2} & 0 & 0 \\
0 & \sigma_{y}^{2} \Delta t+\sigma_{\dot{y}}^{2} \frac{\Delta t^{3}}{3} & 0 & 0 & \sigma_{\dot{y}}^{2} \frac{\Delta t^{2}}{2} & 0 \\
0 & 0 & \sigma_{z}^{2} \Delta t+\sigma_{\dot{z}}^{2} \frac{\Delta t^{3}}{3} & 0 & 0 & \sigma_{\dot{z}}^{2} \frac{\Delta t^{2}}{2} \\
\sigma_{\dot{x}}^{2} \frac{\Delta t^{2}}{2} & 0 & 0 & \sigma_{\dot{x}}^{2} \Delta t & 0 & 0 \\
0 & \sigma_{\dot{y}}^{2} \frac{\Delta t^{2}}{2} & 0 & 0 & \sigma_{\dot{y}}^{2} \Delta t & 0 \\
0 & 0 & \sigma_{\dot{z}}^{2} \frac{\Delta t^{2}}{2} & 0 & 0 & \sigma_{\dot{z}}^{2} \Delta t
\end{array}\right]}
\end{aligned}
$$

where $\Delta t=t_{i}-t_{i-1}$ is the observation interval, and the diagonal elements of matrix $Q(t)$ are determined by trial and error for different circumstances. 


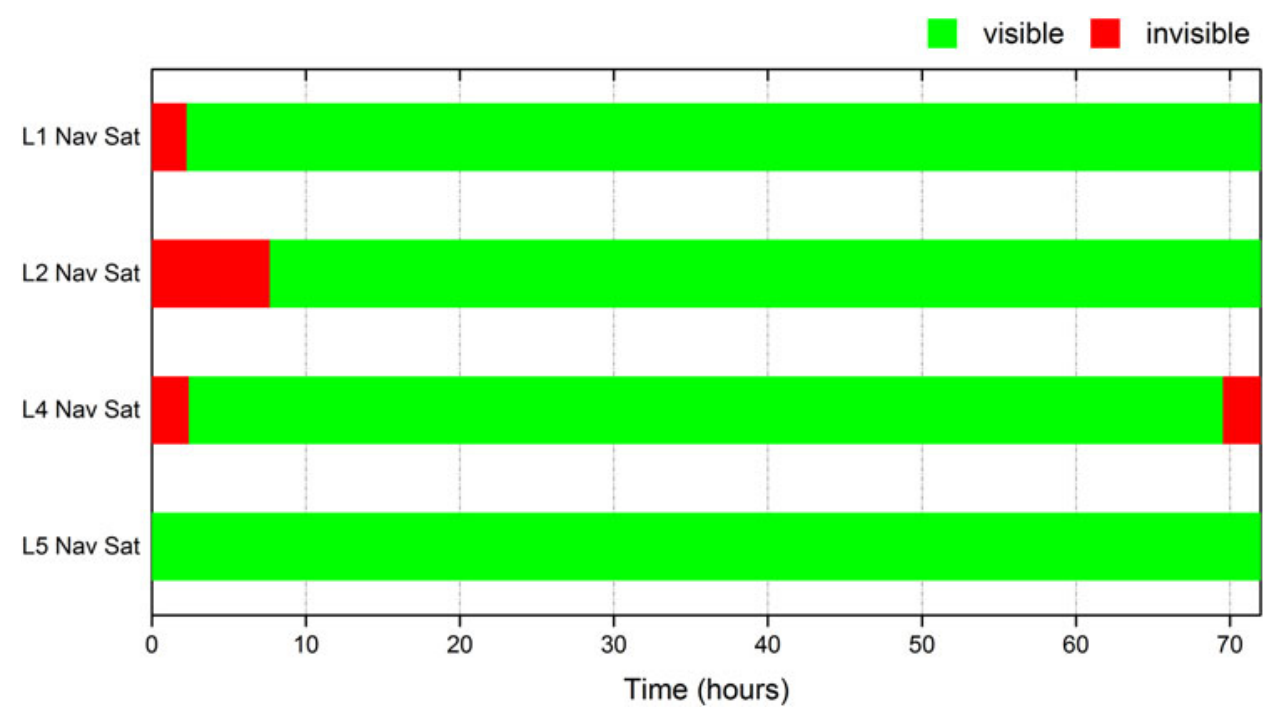

Figure 1. Timeline of visible libration point satellites during the trans-lunar cruise phase. The computation is conducted for the Halo-Halo-VP-VP constellation $(\mathrm{ID}=1)$, while for the other constellations, the results are similar.

\section{NUMERICAL RESULTS OF CISLUNAR NAVIGATION} SIMULATIONS. According to the mission scenarios described previously, a numerical simulation is conducted in this section to study the cislunar navigation performance of the libration point satellite navigation system.

5.1. Trans-Lunar Cruise Navigation Performance. As a starting point, the translunar cruise phase is studied. Based on the dynamical model discussed earlier in Section 3, the nominal trajectory is generated for the user spacecraft. Then an observability analysis is performed for the trans-lunar cruise trajectory using the Earth-Moon $L_{1,2,4,5}$ four-satellite constellations. An illustration of the timeline of visible libration point satellites is shown in Figure 1.

It can be seen from Figure 1 that the spacecraft is visible to the $L_{1}, L_{2}, L_{4}$ navigation satellites for most of the trans-lunar cruise phase, while the $L_{5}$ navigation satellite is always in the field of view for the user spacecraft. Since there is at least one available libration point satellite for the whole trans-lunar cruise trajectory, a navigation performance analysis can then be conducted. Using the nominal orbits of the libration point navigation satellites and the user, a series of satellite-to-satellite range observations are generated every 60 seconds. Considering the ephemeris error of the libration point satellites, a $\sigma_{\text {bias }}=5 \mathrm{~m}$ systematic bias is used to corrupt the observation data. Besides that, a $\sigma_{\text {noise }}=3 \mathrm{~m}$ white-noise error is added to compensate for the stochastic error in every range measurement.

After generating the observation data, the EKF is then employed to estimate the states of the spacecraft. The initial state used to start the filter process is computed from the true trajectory, with an initial position error in each component of $100 \mathrm{~m}$ and an initial velocity error in each component of $0.01 \mathrm{~m} / \mathrm{s}$. The diagonal elements of the process noise covariance matrix $Q(t)$ are determined by trial and error and finally 

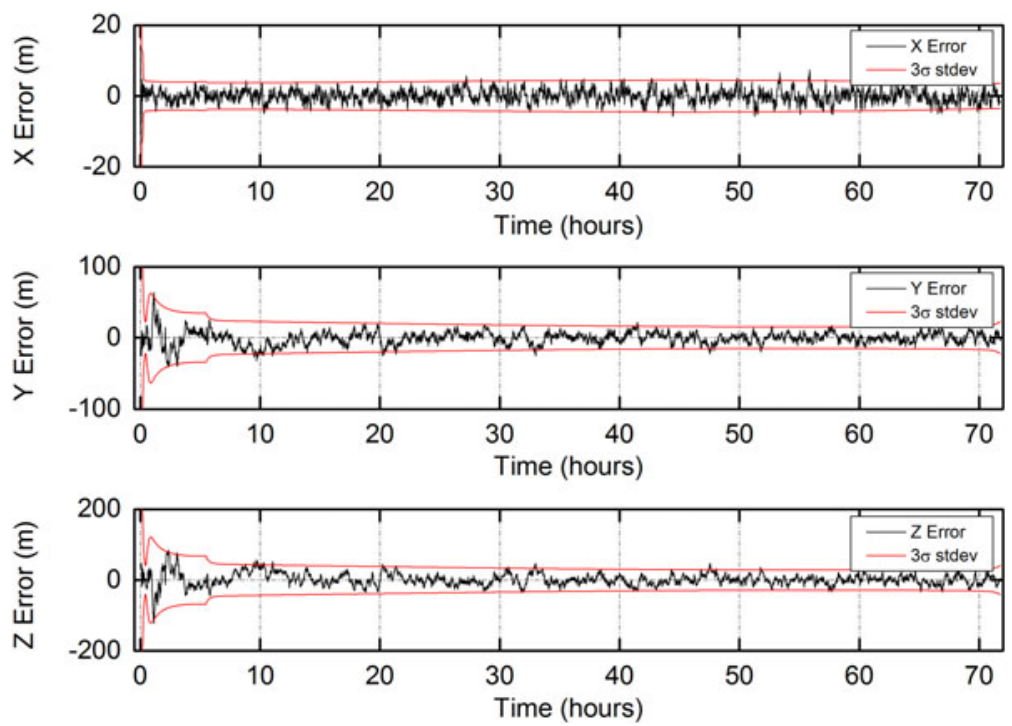

Figure 2. Position estimate error for the trans-lunar cruise phase using the EKF.
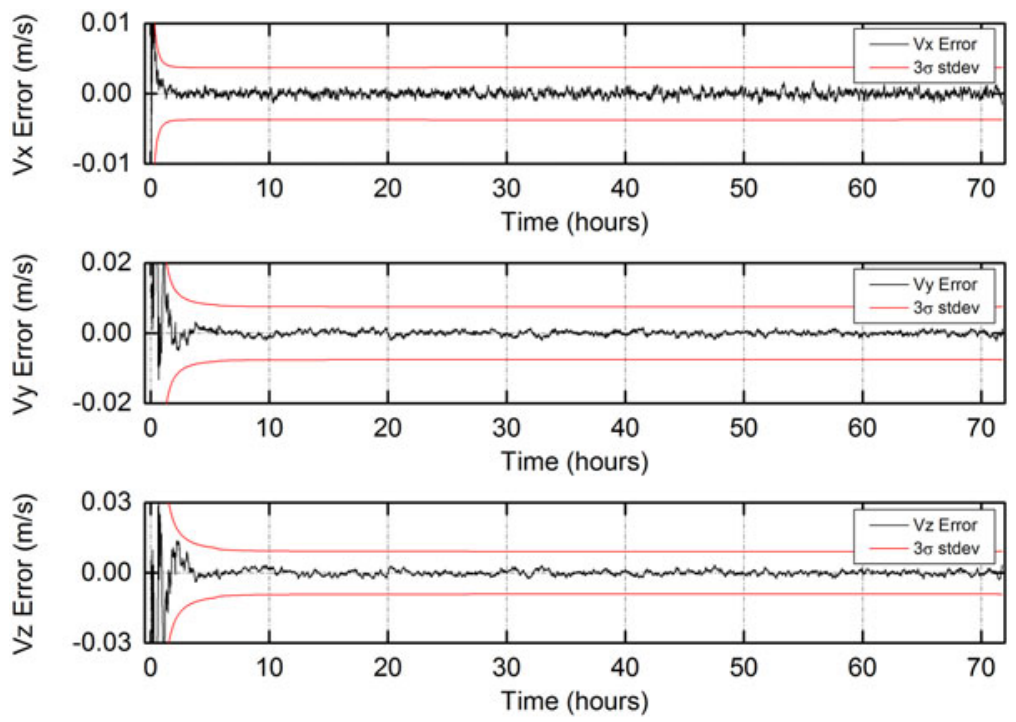

Figure 3. Velocity estimate error for the trans-lunar cruise phase using the EKF.

selected as follows for the trans-lunar cruise phase

$$
\begin{aligned}
& \sigma_{x}^{2}=5.07 \times 10^{-3} \mathrm{~m}^{2} / \mathrm{s}, \sigma_{y}^{2}=6.39 \times 10^{-2} \mathrm{~m}^{2} / \mathrm{s}, \sigma_{z}^{2}=0.11 \mathrm{~m}^{2} / \mathrm{s} \\
& \sigma_{\dot{x}}^{2}=3.98 \times 10^{-10} \mathrm{~m}^{2} / \mathrm{s}^{3}, \sigma_{\dot{y}}^{2}=5.63 \times 10^{-10} \mathrm{~m}^{2} / \mathrm{s}^{3}, \sigma_{\dot{z}}^{2}=6.67 \times 10^{-10} \mathrm{~m}^{2} / \mathrm{s}^{3}
\end{aligned}
$$

Then a trans-lunar cruise navigation simulation is conducted for the candidate EarthMoon $L_{1,2,4,5}$ four-satellite constellations given in Table 1 . The navigation results for the Halo-Halo-VP-VP constellation $(I D=1)$ are shown in Figures 2 and 3, respectively 
Table 4. Trans-lunar cruise navigation performance of the candidate Earth-Moon $L_{1,2,4,5}$ four-satellite constellations.

\begin{tabular}{|c|c|c|c|c|c|}
\hline ID & $\begin{array}{l}\text { Constellation } \\
\text { Type }\end{array}$ & $\begin{array}{l}\text { RMS Position } \\
\text { Error }\end{array}$ & $\begin{array}{l}\text { RMS Velocity } \\
\text { Error }\end{array}$ & $\begin{array}{c}\text { Maximum Position } \\
\text { Error }\end{array}$ & $\begin{array}{c}\text { Maximum Velocity } \\
\text { Error }\end{array}$ \\
\hline 1 & Halo-Halo-VP-VP & $20 \cdot 0 \mathrm{~m}$ & $4.9 \mathrm{~mm} / \mathrm{s}$ & $66.4 \mathrm{~m}$ & $9 \cdot 6 \mathrm{~mm} / \mathrm{s}$ \\
\hline 2 & PL-Halo-VP-VP & $98.5 \mathrm{~m}$ & $10.9 \mathrm{~mm} / \mathrm{s}$ & $216 \cdot 2 \mathrm{~m}$ & $18.8 \mathrm{~mm} / \mathrm{s}$ \\
\hline 3 & VL-Halo-VP-VP & $19 \cdot 5 \mathrm{~m}$ & $4.6 \mathrm{~mm} / \mathrm{s}$ & $56 \cdot 0 \mathrm{~m}$ & $7.5 \mathrm{~mm} / \mathrm{s}$ \\
\hline 4 & Halo-PL-VP-VP & $19 \cdot 6 \mathrm{~m}$ & $4.7 \mathrm{~mm} / \mathrm{s}$ & $67 \cdot 0 \mathrm{~m}$ & $7.8 \mathrm{~mm} / \mathrm{s}$ \\
\hline 5 & PL-PL-VP-VP & $104.6 \mathrm{~m}$ & $14.5 \mathrm{~mm} / \mathrm{s}$ & $196 \cdot 3 \mathrm{~m}$ & $19 \cdot 6 \mathrm{~mm} / \mathrm{s}$ \\
\hline 6 & VL-PL-VP-VP & $21.7 \mathrm{~m}$ & $5 \cdot 3 \mathrm{~mm} / \mathrm{s}$ & $60 \cdot 5 \mathrm{~m}$ & $6.2 \mathrm{~mm} / \mathrm{s}$ \\
\hline 7 & Halo-VL-VP-VP & $19 \cdot 4 \mathrm{~m}$ & $5 \cdot 3 \mathrm{~mm} / \mathrm{s}$ & $59 \cdot 8 \mathrm{~m}$ & $8.7 \mathrm{~mm} / \mathrm{s}$ \\
\hline 8 & PL-VL-VP-VP & $96 \cdot 7 \mathrm{~m}$ & $14.6 \mathrm{~mm} / \mathrm{s}$ & $173.0 \mathrm{~m}$ & $19 \cdot 9 \mathrm{~mm} / \mathrm{s}$ \\
\hline 9 & VL-VL-VP-VP & $18 \cdot 5 \mathrm{~m}$ & $5.5 \mathrm{~mm} / \mathrm{s}$ & $63.6 \mathrm{~m}$ & $6 \cdot 2 \mathrm{~mm} / \mathrm{s}$ \\
\hline
\end{tabular}

for the position and velocity components. It can be seen from the results that the estimated position and velocity components converge quickly to the reference values. The root-mean-square (RMS) position error is $20.01 \mathrm{~m}$ and the RMS velocity error is $4.9 \mathrm{~mm} / \mathrm{s}$. The probability of the position estimate error falling inside the $3 \sigma$ line is $97.72 \%, 98.17 \%$ and $98.12 \%$, respectively for the $x, y$ and $z$ components, while the probability for the velocity estimate error falling inside the $3 \sigma$ line is $100 \%$ for each component. In addition, from Figure 2, it can also been seen that the position estimate error in the X-direction is much smaller than the other two directions, and this may be concerned with the geometrical configuration between the libration point navigation satellites and the user spacecraft. Similar simulations are also conducted for the other candidate Earth-Moon $L_{1,2,4,5}$ four-satellite constellations and the final results are summarised in Table 4.

Table 4 lists the RMS position estimate error and velocity estimate error as well as the maximum position error and velocity error for all the candidate Earth-Moon $L_{1,2,4,5}$ four-satellite constellations. From the results, it can be found that the translunar cruise navigation performance is directly related to the constellation architectures of the libration point satellites, especially the orbit of the $L_{1}$ navigation satellite. When the $L_{1}$ navigation satellite is located in the planar Lyapunov orbit (that is, the $\mathrm{ID}=2,5,8$ constellations), the trans-lunar cruise navigation performance seems always worse than the other configurations, with the RMS position error to be about $100 \mathrm{~m}$ and the RMS velocity error about $10 \mathrm{~mm} / \mathrm{s}$. Once the nominal orbit of the $L_{1}$ navigation satellite is changed to Halo orbit or vertical Lyapunov orbit (that is, the $\mathrm{ID}=1,3,4,6,7,9$ constellations), RMS state estimate error can be reduced to about $20 \mathrm{~m}$ and $5 \mathrm{~mm} / \mathrm{s}$, respectively, for position and velocity, and the maximum position and velocity estimate errors are also controlled within $70 \mathrm{~m}$ and $10 \mathrm{~mm} / \mathrm{s}$. As a result, it may be concluded that the navigation architectures with the $L_{1}$ navigation satellite locating in Halo or vertical Lyapunov orbits are more suitable for trans-lunar cruise navigation.

5.2. Lunar Orbit Navigation Performance. After discussing the trans-lunar cruise navigation performance of the proposed system, a lunar orbit navigation simulation is conducted to further verify the navigation ability in cislunar space. As it has been proved in our previous work that the candidate Earth-Moon $L_{1,2,4,5}$ four-satellite constellations can achieve continuous global coverage for lunar orbits with arbitrary inclination and orbital altitude changing in the range of $100 \mathrm{~km}$ to $2000 \mathrm{~km}$, the lunar 


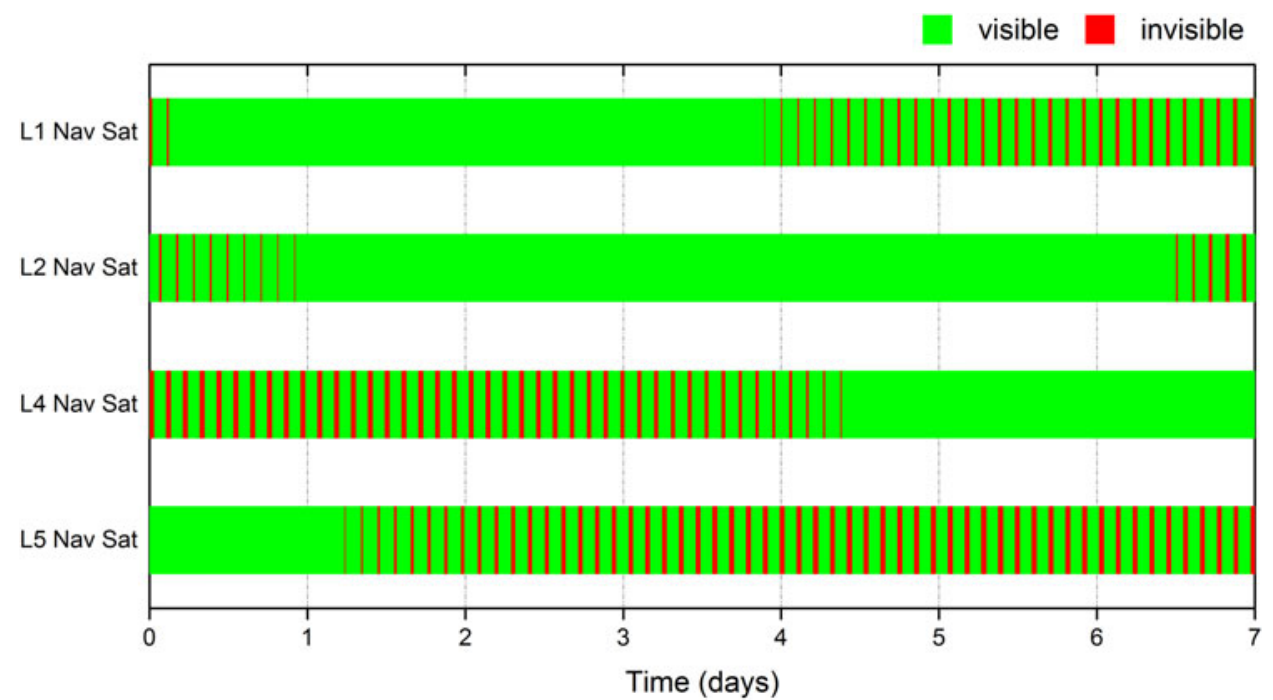

Figure 4. Timeline of visible libration point satellites during the lunar orbit phase. The computation is conducted for the Halo-Halo-VP-VP constellation $(\mathrm{ID}=1)$, while for the other constellations, the results are similar.

orbiter considered in this work can always been tracked by the libration point satellites. An illustration of the timeline of visible navigation satellites in a seven-day period is shown in Figure 4.

It can be seen from Figure 4 that the lunar orbiter is tracked by the four libration point satellites alternately, and there are at least two visible navigation satellites in the seven-day period. For a longer time period, the results are similar and the libration point satellite navigation system is always available to a lunar orbiter. Therefore, a lunar orbit navigation simulation can be conducted. Using the nominal orbits of the user and libration point navigation satellites, a series of scalar satellite-to-satellite range data are generated every 60 seconds with the same systematic bias and whitenoise error as the trans-lunar cruise case. The initial state error considered in the filter process is also $100 \mathrm{~m}$ and $0.01 \mathrm{~m} / \mathrm{s}$ respectively for position and velocity, added to each component of the true state. The process noise covariance matrix $Q(t)$ is determined by trial and error and the diagonal elements are finally selected as follows for the lunar orbit phase

$$
\begin{aligned}
& \sigma_{x}^{2}=2.94 \times 10^{-4} \mathrm{~m}^{2} / \mathrm{s}, \sigma_{y}^{2}=3.70 \times 10^{-3} \mathrm{~m}^{2} / \mathrm{s}, \sigma_{z}^{2}=6.31 \times 10^{-3} \mathrm{~m}^{2} / \mathrm{s} \\
& \sigma_{\dot{x}}^{2}=1.40 \times 10^{-11} \mathrm{~m}^{2} / \mathrm{s}^{3}, \sigma_{\dot{y}}^{2}=1.98 \times 10^{-11} \mathrm{~m}^{2} / \mathrm{s}^{3}, \sigma_{\dot{z}}^{2}=2.35 \times 10^{-11} \mathrm{~m}^{2} / \mathrm{s}^{3}
\end{aligned}
$$

Using the observation data and initial state generated in the above way, a lunar orbit navigation simulation is then conducted for the candidate Earth-Moon $L_{1,2,4,5}$ foursatellite constellations. The navigation results for the Halo-Halo-VP-VP constellation $(\mathrm{ID}=1)$ are shown in Figures 5 and 6 respectively for the position and velocity components. It can be seen from the results that the filter also converges quickly to the steady state. The RMS position error is $23.35 \mathrm{~m}$ and the RMS velocity error is $20.3 \mathrm{~mm} / \mathrm{s}$. The probability of the position estimate error falling inside the $3 \sigma$ line is 

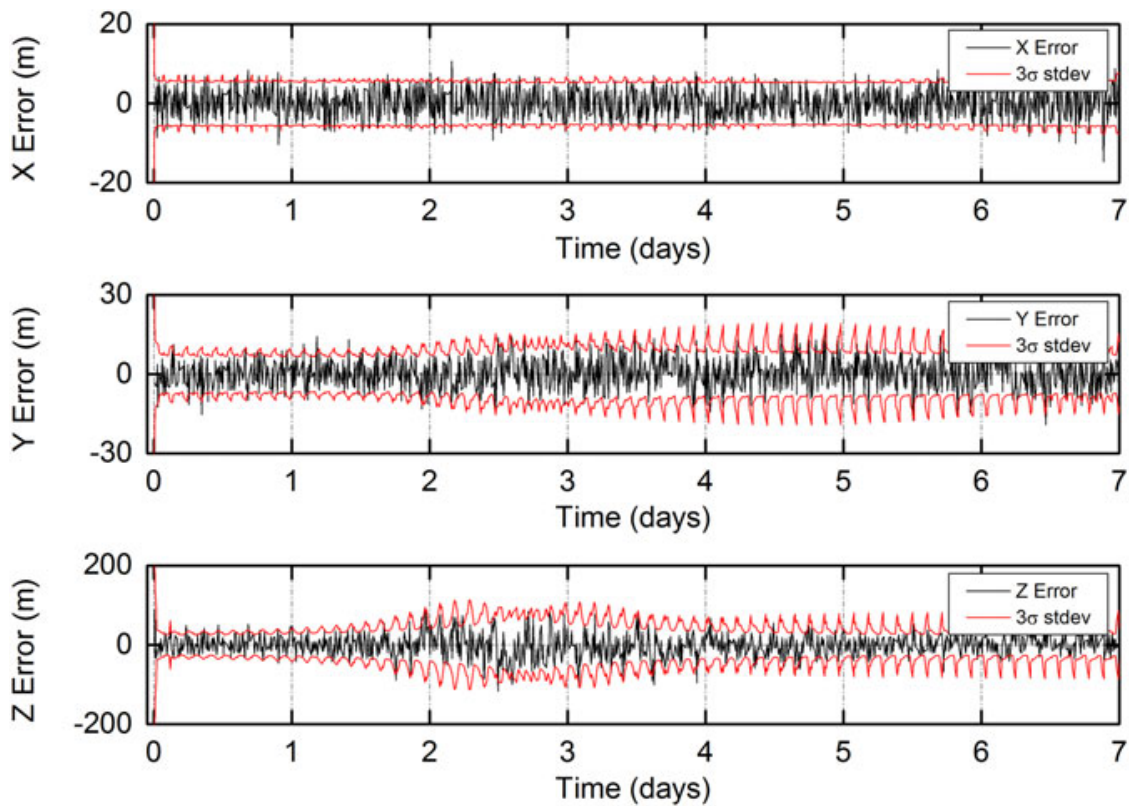

Figure 5. Position estimate error for the lunar orbit phase using the EKF.
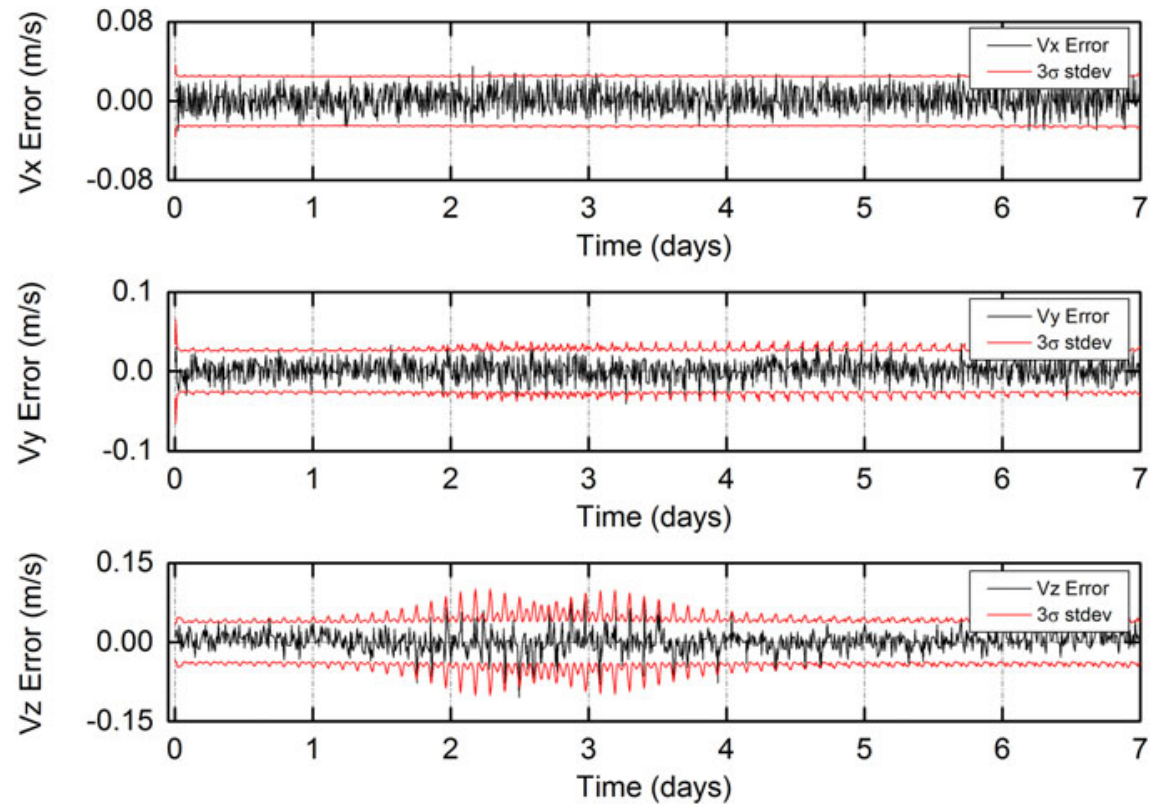

Figure 6. Velocity estimate error for the lunar orbit phase using the EKF.

$97.14 \%, 97.70 \%$ and $97.26 \%$ respectively for the $x, y$ and $z$ components, while the probability for the velocity estimate error falling inside the $3 \sigma$ line is $99.65 \%, 99.61 \%$ and $99.65 \%$ respectively for the $\dot{x}, \dot{y}$ and $\dot{z}$ components. In addition, it can be seen from Figure 5 that the position estimate error in the Z-direction is much larger than 
Table 5. Lunar orbit navigation performance of the candidate Earth-Moon $L_{1,2,4,5}$ four-satellite constellations.

\begin{tabular}{llcccc}
\hline ID & $\begin{array}{l}\text { Constellation } \\
\text { Type }\end{array}$ & $\begin{array}{c}\text { RMS Position } \\
\text { Error }\end{array}$ & $\begin{array}{c}\text { RMS Velocity } \\
\text { Error }\end{array}$ & $\begin{array}{c}\text { Maximum Position } \\
\text { Error }\end{array}$ & $\begin{array}{c}\text { Maximum Velocity } \\
\text { Error }\end{array}$ \\
\hline 1 & Halo-Halo-VP-VP & $23 \cdot 4 \mathrm{~m}$ & $20 \cdot 3 \mathrm{~mm} / \mathrm{s}$ & $117 \cdot 9 \mathrm{~m}$ & $106 \cdot 3 \mathrm{~mm} / \mathrm{s}$ \\
2 & PL-Halo-VP-VP & $33 \cdot 9 \mathrm{~m}$ & $25 \cdot 4 \mathrm{~mm} / \mathrm{s}$ & $146 \cdot 5 \mathrm{~m}$ & $114 \cdot 1 \mathrm{~mm} / \mathrm{s}$ \\
3 & VL-Halo-VP-VP & $25 \cdot 2 \mathrm{~m}$ & $20 \cdot 5 \mathrm{~mm} / \mathrm{s}$ & $124 \cdot 4 \mathrm{~m}$ & $106 \cdot 3 \mathrm{~mm} / \mathrm{s}$ \\
4 & Halo-PL-VP-VP & $23 \cdot 7 \mathrm{~m}$ & $20 \cdot 6 \mathrm{~mm} / \mathrm{s}$ & $113.5 \mathrm{~m}$ & $104 \cdot 5 \mathrm{~mm} / \mathrm{s}$ \\
5 & PL-PL-VP-VP & $34 \cdot 5 \mathrm{~m}$ & $25 \cdot 4 \mathrm{~mm} / \mathrm{s}$ & $144 \cdot 8 \mathrm{~m}$ & $134 \cdot 4 \mathrm{~mm} / \mathrm{s}$ \\
6 & VL-PL-VP-VP & $25 \cdot 4 \mathrm{~m}$ & $20 \cdot 9 \mathrm{~mm} / \mathrm{s}$ & $125 \cdot 4 \mathrm{~m}$ & $99 \cdot 0 \mathrm{~mm} / \mathrm{s}$ \\
7 & Halo-VL-VP-VP & $21 \cdot 8 \mathrm{~m}$ & $19 \cdot 8 \mathrm{~mm} / \mathrm{s}$ & $112.6 \mathrm{~m}$ & $94 \cdot 6 \mathrm{~mm} / \mathrm{s}$ \\
8 & PL-VL-VP-VP & $34 \cdot 0 \mathrm{~m}$ & $24 \cdot 6 \mathrm{~mm} / \mathrm{s}$ & $149 \cdot 3 \mathrm{~m}$ & $130 \cdot 7 \mathrm{~mm} / \mathrm{s}$ \\
9 & VL-VL-VP-VP & $24 \cdot 4 \mathrm{~m}$ & $20 \cdot 8 \mathrm{~mm} / \mathrm{s}$ & $122.2 \mathrm{~m}$ & $92 \cdot 3 \mathrm{~mm} / \mathrm{s}$ \\
\hline
\end{tabular}

the other two directions, which is also concerned with the geometrical configuration of the libration point satellite navigation system. Similar simulations are also conducted for the other candidate Earth-Moon $L_{1,2,4,5}$ four-satellite constellations and the final results of RMS state estimate error and maximum state estimate error are summarized in Table 5.

Similar to the trans-lunar cruise phase, the lunar orbit navigation performance is also sensitive to the orbit of the $L_{1}$ navigation satellite. When the $L_{1}$ navigation satellite is located in the Halo orbit or vertical Lyapunov orbit (that is, the ID $=1,3,4$, $6,7,9$ constellations), the navigation performance is always better, with the RMS position error about $23 \mathrm{~m}$ and the RMS velocity error about $20 \mathrm{~mm} / \mathrm{s}$. But when the orbit of the $L_{1}$ navigation satellite is changed to the planar Lyapunov orbit (that is, the $\mathrm{ID}=2,5,8$ constellations), the lunar orbit navigation performance gets worse, with the RMS position error about $34 \mathrm{~m}$ and the RMS velocity error about $25 \mathrm{~mm} / \mathrm{s}$. Besides that, the maximum state estimate error is also larger for the latter case. Therefore, we can come to the conclusion that the proper Earth-Moon $L_{1,2,4,5}$ foursatellite constellations for cislunar navigation are the ones with the $L_{1}$ navigation satellite locating in Halo or vertical Lyapunov orbits.

6. CONCLUSIONS. In this paper, a virtual lunar exploration mission scenario is developed to verify the cislunar navigation performance of the candidate EarthMoon $L_{1,2,4,5}$ four-satellite constellations proposed in our previous work. The results indicate that the navigation accuracy of a few tens of metres can be achieved for both the trans-lunar cruise and lunar orbit phase. In particular, the Earth-Moon $L_{1,2,4,5}$ four-satellite constellations with the $L_{1}$ navigation satellite locating in Halo orbits or vertical Lyapunov orbits are more suitable for cislunar navigation. Consequently, the proposed libration point satellite navigation system could be available for cislunar navigation and perhaps play an important role in future lunar exploration missions. The navigation performance of our proposed libration point satellite navigation system for Mars explorations will be considered in the following work.

\section{ACKNOWLEDGMENTS}

This work was carried out with financial support from the National Basic Research Program 973 of China (2013CB834103), the National High Technology Research and Development 
Program 863 of China (2012AA121602), and the National Natural Science Foundation of

China (Grant No. 11078001).

\section{REFERENCES}

Bowers, E. (1966). Requirements for onboard optical guidance of spacecraft on lunar trajectories. Journal of Spacecraft and Rockets, 3(3), 328-334.

Christian, J.A. and Lightsey, E.G. (2009). Review of Options for Autonomous Cislunar Navigation. Journal of Spacecraft and Rockets, 46(5), 1023-1036.

Defense Mapping Agency. (1984). Technical Report. Department of Defense World Geodetic System 1984, DMA TR 8350.2, Second Ed.

Farquhar, R.W. (1967). Lunar communications with libration-point satellites. Journal of Spacecraft and Rockets, 4(10), 1383-1384.

Hill, K., Born, G.H., and Lo, M.W. (2005). Linked, Autonomous, Interplanetary Satellite Orbit Navigation (LiAISON) in Lunar Halo Orbits. Paper AAS 05-400, AAS/AIAA Astrodynamics Specialist Conference, Lake Tahoe, CA.

Hill, K. (2007). Autonomous Navigation in Libration Point Orbits. Ph.D. thesis, Graduate School of the University of Colorado.

IAU SOFA Board. (2010). IAU SOFA Software Collection. Issue 2010-12-01, available at http://www. iausofa.org.

Keenan, R. and Regenhardt, J. (1962). Star Occultation Measurements as an Aid to Navigation in Cis-Lunar Space. M.S. Dissertation, Massachusetts Inst. of Technology, Cambridge, MA, June.

Konopliv, A.S., Asmar, S.W., Carranza, E., Sjogren, W.L., and Yuan, D.N. (2001). Recent gravity models as a result of the Lunar Prospector mission. Icarus, 150(1), 1-18.

Leonard, J.M., Parker, J.S., Anderson, R.L., McGranaghan, R.M., Fujimoto, K., and Born, G.H. (2013). Supporting crewed lunar exploration with LiAISON navigation. Paper AAS 13-053, In 36th Annual Guidance and Control Conference, Breckenridge, Colorado.

Lei, H. L., Xu, B., Hou, X. Y. and Sun, Y.S. (2013a). High-order solutions of invariant manifolds associated with libration point orbits in the elliptic restricted three-body system. Celestial Mechanics and Dynamical Astronomy, 117(4), 349-384.

Lei, H. L. and Xu, B. (2013b). High-order analytical solutions around triangular libration points in the circular restricted three-body problem. Monthly Notices of the Royal Astronomical Society, 434. 2.

Liu, L. and Wang, X. (2006). An Orbital Dynamics of Lunar Probe [M]. Beijing: National Defense Industry Press. (in Chinese).

Montenbruck, O. and Gill, E. (2000). Satellite Orbits: Models, Methods, and Applications. Springer Verlag, Heidelberg, Germany.

Parker, J.S., Leonard, J. M., Fujimoto, K., McGranaghan, R.M., Born, G.H., and Anderson, R.L. (2013). Navigating a Crewed Lunar Vehicle Using LiAISON. Paper AAS 13-330, 23rd AAS/AIAA Space Flight Mechanics Meeting, Kauai, Hawaii.

Standish, E.M. (1998). JPL Planetary and Lunar Ephemerides, DE405/LE405. JPL IOM 312.F-98-048.

Tuckness, D.G. and Young, S.Y. (1995). Autonomous navigation for lunar transfer. Journal of Spacecraft and Rockets, 32(2), 279-285.

Tapley, B.D., Schutz, B.E., and Born, G.H. (2004). Statistical orbit determination (Vol. 200). Burlington: Elsevier Academic Press.

US Nautical Almanac Office. (2013). The Astronomical Almanac for the Year 2014. Government Printing Office.

Zhang, L. and Xu, B. (2014). A Universe Light House - Candidate Architectures of the Libration Point Satellite Navigation System. Journal of Navigation, 67(5), 737-752. 